\title{
Linx
}

Revue des linguistes de l'université Paris X Nanterre

40 | 1999

Le statut d'unité lexicale

\section{Termes et locuteurs}

\section{Marie-Françoise Mortureux}

\section{(2) OpenEdition \\ Journals}

Édition électronique

URL : http://journals.openedition.org/linx/737

DOI : $10.4000 /$ linx.737

ISSN : 2118-9692

\section{Éditeur}

Presses universitaires de Paris Nanterre

\section{Édition imprimée}

Date de publication : 1 juin 1999

Pagination : $33-53$

ISSN : 0246-8743

\section{Référence électronique}

Marie-Françoise Mortureux, "Termes et locuteurs », Linx [En ligne], 40 | 1999, mis en ligne le 20 juin

2012, consulté le 19 avril 2019. URL : http://journals.openedition.org/linx/737 ; DOI : 10.4000/linx.737

Ce document a été généré automatiquement le 19 avril 2019

Département de Sciences du langage, Université Paris Ouest 


\title{
Termes et locuteurs
}

\author{
Marie-Françoise Mortureux
}

\section{Les termes : une catégorie particulière de mots?}

1 L'existence d'une catégorie particulière de mots, appelée termes, est une opinion largement répandue. Cependant, les propriétés des termes ne sont pas toujours clairement et unanimement reconnues; et leur usage soulève fréquemment des polémiques: la terminologie, entendue d'abord comme ensemble de termes (Kocourek 1982), est ressentie par les divers locuteurs tantôt comme un jargon inutile et irritant, tantôt comme un moyen de communication indispensable.

2 Le propos ici est d'examiner la question à la lumière de deux expériences différentes qu'on peut avoir des termes: d'une part, l'expérience courante que tout locuteur en acquiert dans les échanges ordinaires, c'est-à-dire en dehors d'une activité spécifique, et d'autre part la compétence en terminologie que possèdent certains spécialistes dans le cadre d'une activité déterminée.

\section{L'expérience courante}

Une démarche élémentaire et disponible à tout locuteur alphabétisé consiste à ouvrir un dictionnaire de langue pour savoir ce qu'est un terme.

\section{1. « terme » dans les dictionnaires de langue}

4 De fait, les termes reçoivent dans les dictionnaires de langue des définitions qui caractérisent ces mots, comme on peut le voir, par exemple dans le PRE : 
Terme n. $\mathbf{m}$

-v. 1050; lat. terminus, proprt « borne»

II (1370; lat. médiév. terminus « ce qui limite le sens »)

$1 \diamond$ Mot ou expression.

$2 \checkmark$ Mot appartenant à un vocabulaire spécial, qui n'est pas d'un usage courant dans la langue commune.

5 Cette définition caractérise les termes sur un critère sociolinguistique «vocabulaire spécial »; elle demeure minimale, se contentant d'en exclure l'occurrence de «l'usage courant commun ».

6 Un autre dictionnaire de langue moins répandu, le Lexis, proposait un autre critère, faisant du terme un « mot qui a un sens strictement délimité à l'intérieur d'un système de notions".

7 Deux traits, l'un sociolinguistique, l'autre sémiotique, sont donc alternativement retenus pour caractériser ces mots. Ils correspondent sans doute à l'intuition de la plupart des locuteurs, qui, de ce fait, ne s'attendent pas à trouver des termes n'importe où...

\section{Rencontres avec des termes}

8 Or, il est de fait qu'on rencontre des termes au détour de lectures ou de conversations, sous l'apparence de mots ordinaires ou de mots étranges, dont l'interprétation peut ou non embarrasser, selon les cas.

9 Pour commencer, voici quelques extraits de textes d'origine variée à l'occasion desquels se pose la question des termes, de leur repérage, et de leur interprétation. Ils proposent, de façon un peu schématique, un éventail de possibilités diverses, allant de la méconnaissance totale du terme à une reconnaissance doublée d'une difficulté d'interprétation. Le point commun aux discours auxquels sont empruntés ces extraits est de concerner un public assez large, qu'on ne peut pas assimiler à un cercle étroit de spécialistes.

\section{Budget}

10 Tout le monde aujourd'hui peut entendre parler du vote du budget par l'Assemblée Nationale, comme on peut aussi se fixer un budget vacances. Et personne, sans doute, n'est embarrassé par ce mot. Cependant, dans le premier cas, on a bien affaire à un terme, créé en même temps que fut instituée la procédure qui le définit : « Acte par lequel sont prévues et autorisées les recettes et les dépenses annuelles de l'Etat » (décret du 5 mai 1862). Le nom budget, emprunté à l'anglais, est d'abord un terme de droit public. Aujourd'hui, il peut aussi désigner couramment une somme d'argent disponible pour une dépense quelconque.

\section{Tourbillon}

11 Pour apprécier l'effet comique recherché par Molière lorsqu'il met dans la bouche de Trissotin les propos suivants : 

donné par Descartes à ce néologisme n'ajoute guère au comique, et c'en est à peine plus
drôle si l'on comprend que leur visiteur annonce ainsi aux Femmes savantes le passage d'une comète.

\section{Neutrino}

15 En revanche, un minimum de connaissance des concepts de la physique contemporaine est nécessaire pour comprendre la phrase titre d'un article de première page dans le journal Le Monde :

Les neutrinos ont une masse et la physique est en révolution. (Le Monde, 7-8/06/98).

16 A tout le moins faut-il savoir que les neutrinos sont des particules, et que leur masse est un objet de recherche contemporain, comme l'indiquent ces extraits d'ouvrages de vulgarisation rédigés l'un et l'autre en 1988 par deux spécialistes, dont le premier, astrophysicien, écrit en français, et le second, physicien, écrit en allemand et a été récemment traduit en français :

La libération de l'énergie du vide dans l'univers pendant la phase inflationnaire va avoir une autre conséquence extrêmement importante. Elle va donner naissance au contenu matériel de l'univers. [...] les quarks, les électrons, les neutrinos et leurs antiparticules vont surgir du vide. (Trinh Xuan Than, La mélodie secrète, Paris, Fayard, 1988, p.153).

Il y a d'ailleurs très vraisemblablement dans la nature, mis à part les photons, les particules de lumière, d'autres particules qui se meuvent toujours à la vitesse de la lumière, les neutrinos. Ce sont des particules électriquement neutres, apparentées aux électrons. Ce sont en quelque sorte les frères neutres de l'électron, créés au cours de certaines réactions nucléaires. [...] «très vraisemblablement » [...] parce qu'on ne sait toujours pas, à ce jour, si ces neutrinos, comme les photons, n'ont pas de masse mais une énergie déterminée, ou s'ils en possèdent une, même très petite. Dans ce dernier cas, ils ne peuvent jamais se déplacer à la vitesse de la lumière, à l'instar des protons du CERN. (Harald Fritsch, $\mathrm{E}=\mathrm{mc}^{2}$ une formule change le monde, trad. Paris, O. Jacob, 1998, éd. originale Piper Verlag, Munich, 1988).

Il s'agit cette fois-ci d'un terme clairement associé au domaine de la physique contemporaine, désignant une particule, qui a reçu un nom créé en même temps que le concept correspondant, et motivé dans le sous système terminologique des particules. Son repérage, facilité par la morphologie, est facile ; son interprétation l'est moins : elle dépend du contexte discursif, plus ou moins éclairant selon les cas, comme on peut le constater à travers ces exemples.

\section{Qu'est-ce qu'une race?}

18 Les deux extraits suivants sont également empruntés au journal Le Monde. Le premier relève des informations, le second du courrier des lecteurs ; leur seul point commun est la mise en cause du mot race :

Le tribunal correctionnel de Dijon a estimé, mercredi 10 décembre, que la dénonciation par un professeur des "hordes musulmanes" ne tombait pas sous le coup de la provocation à la haine raciale et de l'insulte raciale car les musulmans « ne constituent pas une race particulière mais se rencontrent parmi des peuples variés ॥. [...] 
- [Le professeur] évoquait le débarquement en France de "hordes musulmanes inassimilables » qui « ont investi les plus reculés de nos cantons ». (Le Monde, 12/12/97).

« Race » catalane. Fidèle lecteur de votre titre et amoureux du ballon ovale, je me permets de vous écrire pour vous faire part de mon étonnement en entendant à plusieurs reprises les commentateurs sportifs de France 2 vanter les « vertus » de la " race catalane ", lors de la finale du Championnat de rugby à XV.

Ces termes inexacts et qui hélas entraînent trop souvent de fâcheux dérapages, avaient-ils leur place sur une chaîne publique à une heure de grande écoute ? (Le Monde TRM, 01 au 07/06/98).

19 Dans les deux cas, la désignation d'un groupe humain par le mot race est présentée comme une possible insulte, soit par implication négative dans le jugement rendu par le tribunal, soit par un sous-entendu dans la lettre de lecteur (le terme est explicitement qualifié d'« inexact » et jugé responsable de «fâcheux dérapages »). Que signifie donc le mot race?

\section{Et le patoutisme?}

Quant à ce troisième exemple de terme, il vient au détour d'une communication présentée aux Journées de l'Association Freudienne Internationale au Centre Hospitalier Sainte Anne à Paris les 14 et 15 juin 1997. Ces journées rassemblaient linguistes, psychanalystes et professionnels de l'écrit (un correcteur notamment) sur le thème de la ponctuation.

pas tout $\mathbf{x}$ phi de $\mathbf{x}$ : formule du patoutisme avec cette petite barre sur le quanteur à laquelle Lacan nous propose de substituer l'aleph 0 , signe du dénombrable déjà apparu dans ce séminaire unique des Noms du Père du 20 novembre 1963, «aleph de l'angoisse, ce à quoi se rassemble dans sa structure la plus fondamentale le rapport du sujet $a u$ a ». (Le discours psychanalytique, revue de l'Association freudienne, $n^{\circ} 18$, octobre 1997) ${ }^{1}$.

21 Il apparaît fort obscur au non psychanalyste, voire au non lacanien, puisque la définition proposée emprunte les concepts lacaniens. Mais il se repère, outre ce problème d'interprétation, par son signifiant qui, relié explicitement au "pas tout», en fait un signe motivé.

Ces quelques exemples suffisent à montrer que n'importe qui est exposé aux termes en toute sorte d'occasions. Non seulement dans son domaine propre d'activité, dont le vocabulaire lui est familier, mais au hasard de ses lectures (voire de ses conversations), sans que nécessairement il prenne toujours clairement conscience du phénomène. Les exemples cités ici en sont la preuve: les termes peuvent être méconnus (budget), non compris (tourbillon), ; ils peuvent aussi, s'ils sont repérés (neutrino,) être peu compris, ou bloquer la communication (patoutisme). Parmi les mots qui font obstacle à la communication, race est abusivement pris pour un terme par le correspondant du journal, et, en tant que terme juridique, allègrement confondu avec un autre mot (qui serait religion) par celui qui a intenté une action en justice contre ce "professeur » qui, lui, apparemment maitrisait son vocabulaire! Patoutisme figure dans un discours qui, bien qu'il ne soit pas destiné aux seuls lacaniens, ne manifeste guère de souci vulgarisateur à l'adresse des linguistes et professionnels de l'écrit coparticipants du colloque.

23 Les termes sont partout, et on ne les voit pas toujours là où ils sont : telles sont les deux informations illustrées jusqu'ici. Elles permettent de comprendre un aspect de la polémique dont ils sont l'objet, car la difficulté d'interprétation qu'ils peuvent provoquer 
suscite l'agacement des récepteurs, contraints de reconnaître leur insuffisante compétence.

Par ailleurs, on remarque que ni sous l'aspect morphologique ni sous l'aspect sémantique, les exemples proposés ne manifestent d'homogénéité susceptible d'en faire une catégorie particulière de mots.

\section{Représentation lexicographique des termes}

Pour comprendre les mots embarrassants (termes ou non), là encore on peut recourir aux dictionnaires. Quels ouvrages enregistrent les termes? Comment les traitent-ils? Ce traitement manifeste-t-il la spécificité des termes?

Certains termes sont enregistrés dans les dictionnaires de langue courants (type Petit Robert), d'autres n'apparaissent que dans des recueils spécialisés. Couramment, on a surtout accès aux dictionnaires de langue et aux encyclopédies.

\section{Dictionnaire de langue}

27 Les termes cités ici sont traités différemment dans le Petit Robert, dont la révision de 1996 a donné lieu à une édition électronique.

\section{Budget}

Aucune mention de domaine n'apparaît dans l'article. La valeur terminologique est définie en premier, assortie de la mention du décret fondateur; et elle est suivie d'une seconde acception, introduite par la mention Par anal. On est surpris de constater que la première acception est datée de 1862, et la seconde de 1801, l'emprunt à l'anglais datant de 1764. L'ordre logique (sens restreint 魔 sen large) l'a-t-il emporté sur la chronologie, ou bien la valeur en anglais a-t-elle été prise en compte?

\section{Tourbillon}

Parmi les cinq acceptions définies, la quatrième est introduite par la mention Hist. Sc. Et présente une définition succincte du concept « système matériel animé d'un mouvement de rotation", exemplifiée par la phrase "Newton ruina la théorie des tourbillons de Descartes ».

1313. Neutrino

Ce terme figure à la nomenclature du PRE :

neutrino $\mathrm{n} . \mathrm{m}$

-1935; mot ital., de neutro «neutre"

- Particule (lepton) électriquement neutre, de masse infime, de spin 1/2, capable de traverser toute matière. Neutrino de l'électron. $\Rightarrow$ aussi antineutrino.

31 De même, le terme auquel il renvoie : 
antineutrino n. m.・1958; de 1 . anti- et neutrino

- Phys. Antiparticule du neutrino.

On peut, de plus, remarquer que la marque de domaine est absente pour le premier et présente pour le second. Ce choix intrigue d'autant plus que le second est clairement motivé relativement au premier...

Pour la masse, qui intervient dans la définition de neutrino, l'article assez long qui lui est consacré comporte une section spéciale correspondant à l'emploi qui figure dans la définition de neutrino:

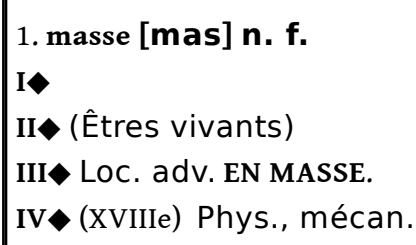

$1 \diamond$ Quantité de matière d'un corps; rapport constant qui existe entre les forces qui sont appliquées à un corps et les accélérations correspondantes.

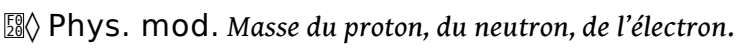

$2 \diamond$ Électr. Masse électrique, magnétique: grandeur sur laquelle un champ (électrique, magnétique) exerce son action pour produire une force.

$\diamond$ Cour. Conducteur commun auquel sont reliés les divers points d'un circuit qui doivent être affectés du même potentiel, en principe celui du sol.

34 Seule la section IV est concernée par l'acception spécialisée en physique. Et elle est introduite par la mention du domaine.

\section{Race}

La consultation du PRE ne permet pas tout d'abord de comprendre les raisons de la « connotation » négative attachée à ce mot, et relevée plus haut : 
race $\mathbf{n} . \mathbf{f}$.

I医

$1 \checkmark$ Famille, considérée dans la suite des générations et la continuité de ses caractères (ne se dit que de grandes familles, familles régnantes, etc.).

$\checkmark \mathrm{V}$ Descendance, postérité.

$2 \checkmark$ Vieilli Communauté plus vaste considérée comme une famille, une lignée.

$3 \triangleleft$ Fig. Catégorie de personnes apparentées par des comportements communs.

II Subdivision de l'espèce zoologique, elle-même divisée en sous-races ou variétés, constituée par des individus réunissant des caractères communs héréditaires.

$\checkmark$ Absolt Race pure.

III (Groupes humains)

$1 \checkmark(1684)$ Groupe ethnique qui se différencie des autres par un ensemble de caractères physiques héréditaires (couleur de la peau, forme de la tête, proportion des groupes sanguins, etc.) représentant des variations au sein de l'espèce.

$2 \triangleleft$ Par ext. (XIXe) Groupe naturel d'hommes qui ont des caractères semblables (physiques, psychiques, culturels, etc.) provenant d'un passé commun.

Aucune de ces acceptions n'est marquée péjorativement. Et l'on n'hésite guère à reconnaitre dans l'expression «race catalane » l'actualisation de la dernière (III 2)... Quant au délit évoqué par le jugement rendu à Dijon («provocation à la haine raciale, insulte raciale »), ce n'est pas dans cet article qu'on peut en trouver l'origine, mais, bien évidemment, dans l'article consacré au dérivé racisme, reproduit intégralement cidessous :

racisme n. m.・1902; de race

1- Théorie de la hiérarchie des races, qui conclut à la nécessité de préserver la race dite supérieure de tout croisement, et à son droit de dominer les autres. Le racisme n'a aucune base scientifique. "Mein Kampf est [...] l'évangile du national-socialisme, ou, plus exactement, du racisme $»$ (Bainville).

$\checkmark$ Ensemble de réactions qui, consciemment ou non, s'accordent avec cette théorie. Faire preuve de racisme. Ligue internationale contre le racisme et l'antisémitisme (L. I. C. R. A.).

$2 \triangleleft$ Abusivt Hostilité violente contre un groupe social. Racisme envers les femmes. $\Rightarrow$ sexisme. Racisme anti-jeunes. "ça a été très compliqué d'entrer dans le cinéma à cause du type à l'entrée qui faisait du racisme anti petits garçons » (‥ Forlani).

L'explication ne repose pas sur le sens du mot, ni même sur une mention quelconque de péjoration; elle tient à la citation de Bainville, qui associe le livre d'Hitler (en allemand) au concept de racisme. De même, l'adjectif racial est constamment défini :

racial, iale, iaux adj.

- Relatif à la race, aux races (III).

Ce qui en soi n'est pas davantage péjoratif.

La péjoration ne tient donc pas à la langue, mais à la culture. 
40 Du moins pas au lexique général. Car il existe une série de délits qui, définis dans le discours juridique, font intervenir le concept de race, à travers la mention de l'adjectif qui en est dérivé : " provocation à la haine raciale, insulte raciale, discrimination raciale ». Il s'agit là d'un emploi spécialisé de l'adjectif, qui, dans ce domaine, a acquis, dans la seconde moitié du XXème siècle, et consécutivement à l'histoire du nazisme, le statut de terme. C'est l'acception numérotée III 1 (dans le PRE) du nom polysémique race qui a été théorisée par le racisme ; et c'est elle aussi qui fonde la notion juridique, invoquée par le tribunal. Mais les acceptions I 3 et III 2, qui ne s'opposent que par le type de la communauté (catégorie ou groupe naturel) fondé sur le comportement ou le partage de caractères eux-mêmes variés, sont vraisemblablement actualisées dans les propos rapportés par Le Monde et cités ci-dessus.

41 Dans un cas comme celui-ci, la confusion entre valeur terminologique et valeur générale menace de disparition, pour des raisons idéologiques, les valeurs générales de race, appliquées à des groupes humains. C'est, bien évidemment, à la notion juridique que réfère la décision du tribunal de Dijon. Et, inversement, ce n'est pas elle qu'évoquent les commentaires sportifs. En revanche, les propos suivants tenus publiquement, fin juin 1998, par un spectateur du Mondial, indigné par le comportement des hooligans, ne fontils pas appel à cette acception condamnée idéologiquement :

Quand on voit les supporters anglais et allemands, et les supporters japonais, on ne peut quand même pas dire qu'il n'y a pas de race supérieure!

Le rapprochement entre ces petits textes et le dictionnaire de langue illustre deux choses : d'abord la fréquente confusion opérée par les locuteurs entre termes et autres vocables ; ensuite, le caractère contraignant des définitions spécialisées, qui restent liées à un domaine déterminé. La mention de domaine, si elle n'est pas absente des dictionnaires de langue, reste cependant la particularité des dictionnaires encyclopédiques.

\section{Et patoutisme?}

43 Ce dernier terme ne figure pas dans les dictionnaires de langue de taille moyenne qui ont été consultés, et dont le PRE est sans nul doute un très bon représentant.

En conclusion, le traitement des termes dans les dictionnaires de langue reflète, autrement, l'embarras des locuteurs à leur égard. De ce point de vue, l'ouvrage choisi ici comme référence est représentatif d'une difficulté générale, qui affecte simultanément le fonctionnement des termes dans les divers discours et leur représentation lexicographique. Tantôt lexème à part entière, avec une entrée séparée, tantôt acception spécialisée d'un lexème "général ", tantôt muni d'une marque de domaine, tantôt dépourvu de cette indication, éventuellement récupérable par référence à un autre terme : cette diversité de traitement peut être reliée aux divers degrés de banalisation des termes, c'est-à-dire à la probabilité pour un locuteur quelconque de rencontrer le mot en dehors des discours strictement spécialisés. Un terme non banalisé (par exemple patoutisme) ne figure pas dans les dictionnaires de langue courants; des termes de grande diffusion (via l'école), comme ceux qui désignent les particules (photon, neutrino) identifiées par la physique atomique, ou des concepts fondamentaux (masse) y sont en général enregistrés, de façon variable, en fonction de leur morphologie, qui en fait soit des homonymes de mots ordinaires (masse), soit des mots dont le signifiant, même dérivé, leur est propre (neutrino). Les premiers posent notamment des problèmes 
redoutables aux lexicographes. Les relations entre soleil et étoile sont exemplaires d'un conflit entre l'usage courant et la valeur scientifique, terminologique, des deux mots; elles provoquent dans un dictionnaire comme le Furetière, qui témoigne à la fin du 17ème siècle de la récente diffusion de l'héliocentrisme dans le grand public, de contradictions insolubles entre valeurs ancienne et nouvelle : le soleil est tantôt associé à la lune (« les deux grands luminaires »), et opposé aux étoiles fixes, tantôt inclus dans les étoiles, type dont il n'est qu'un exemplaire (Mortureux 1979). Ces « conflits » entre deux approches, l'une fondée sur l'expérience naïve et immédiate des locuteurs (structurée par l'opposition entre le jour et la nuit, entre le proche et le lointain) et la connaissance scientifique (informée par une radicale mise en cause de cette expérience) laissent encore des traces dans les ouvrages du 20ème siècle, comme le montre l'analyse du PR par S. Delesalle et A. Rey :

\section{Ainsi, l'assertion :}

(1) le soleil est une étoile

étant vraie, l'assertion :

(2) le soleil empêche de voir les étoiles

devrait être fausse; or, elle est intuitivement vraie; et la phrase assertive :

(3) Cette étoile estle soleil

devrait être sémantiquement acceptable; or, elle ne l'est pas dans lacompétence lexicale générale.

Pour rétablir une description intuitivement correcte, il faut recourir pour étoile à deux sémèmes, dont le premiercorrespond à une descriptionculturelle stéréotypée. " point brillant dans le ciel, la nuit... ", et le second à la construction d'une notion de nature scientifique : " astre producteur d'énergie » (avec des spécifications possibles). Les deux classes d'objets correspondant à ces définitions sont distinctes l'objet «soleil », par exemple, est exclu du premier, inclus dans le second.

De nombreuses classes d'objets de savoir, de nombreux signes sont dans cette situation. (Delesalle et Rey, 1979, p.24).

Le témoignage que donnent sur les termes les dictionnaires de langue apparaît fiable ; il reflète plutôt bien leur place dans la compétence lexicale commune. Ce sont des mots parmi les autres, qui ne forment pas une catégorie homogène : ni leur morphologie, qui n'affecte pas à tous un signifiant original, ni leur sens, tantôt simple spécialisation du sens d'un lexème, tantôt exclusif, ni leur diffusion ne permettent de les définir par opposition au reste du lexique.

\section{Dictionnaires encyclopédiques et encyclopédies}

La différence entre ces deux formules est liée à leur présentation. Le dictionnaire encyclopédique traditionnel se présente davantage comme une liste alphabétique de mots entrées, à l'instar d'un dictionnaire de langue; mais le contenu des articles est essentiellement constitué d'exposés des connaissances liées au concept nommé en entrée dans chacun des domaines auquel il appartient. L'encyclopédie contemporaine, de plus en plus souvent électronique (cédérom, puis DVD), établit un lien entre un mot donné et la liste des articles, des textes cités ou des thèmes dans lesquels il intervient. Par exemple, le mot race est relié, entre autres, au thème " génétique "; et l'exposé sur la génétique des races conclut, via les calculs statistiques sur la répartition des gènes, à l'abandon du concept de race tel qu'il avait été précédemment élaboré, et avait été invoqué par les tenants du racisme (Encyclopédie Hachette Multimédia). De même, le neutrino figure dans divers exposés relevant de la physique... Patoutisme demeure introuvable (même dans les 
dictionnaires spécialisés de psychanalyse, tels les ouvrages de Laplanche et Pontalis, ou d'E. Roudinesco).

On pourrait multiplier les exemples. Cela ne ferait que confirmer globalement les conclusions auxquelles mènent ceux-ci: les termes font partie de notre expérience courante du vocabulaire. Mais ils peuvent perturber la communication. C'est même là le premier « symptôme » auquel on peut reconnaître un terme, bien qu'il ne soit pas fiable, parce que tout vocable ressenti comme obscur par un locuteur quelconque n'est pas forcément un terme. En outre les dictionnaires aisément accessibles fournissent sur ces mots des informations inégalement riches, qui ne suffisent pas toujours à lever la difficulté. Les termes, tout en appartenant à l'expérience générale de tout locuteur, échappent à la compétence lexicale commune. La conjonction de ces deux facteurs explique la condamnation dont ils font parfois l'objet.

\section{La maîtrise des termes}

Au contraire des situations précédentes, le «vocabulaire spécial » $(P R)$ que forment les termes peut apparaître fort clair aux spécialistes qui le créent, le manient et le recueillent.

\section{Définitions spécialisées de «terme »}

Comme nous avons consulté ci-dessus des définitions lexicographiques courantes de ce mot, nous en cherchons maintenant une approche spécialisée.

\section{Une définition de linguiste}

Le linguiste R. Kocourek a consacré au français scientifique et technique une étude qui fait référence. Voici comment il définit le terme :

Le terme est une unité lexicale définie dans les textes de spécialité. L'ensemble des termes s'appelle la terminologie. [...] Les termes sont [...] des unités lexicales dont le sens est défini par les spécialistes dans les textes de spécialité. (Kocourek 1982, p. 77). Ces propositions sont apparemment sans ambiguïté : ce qui fait le terme, c'est sa présentation par certains énonciateurs (autorisés, parce que spécialistes) dans certains discours dits de spécialité.

La terminologie, telle que définie ci-dessus, n'est qu'un vocabulaire particulier à certains groupes sociaux, comme l'affirme le PRE.

Cependant, il faut préciser dès maintenant l'expression « discours de spécialité »; elle se prête à deux interprétations: l'une, restreinte, qui entend par là des discours qui s'adressent à d'autres spécialistes, ceux que les sociologues de la connaissance appellent discours "primaires». L'autre, plus large, y englobe l'ensemble, très abondant et hétérogène dans notre société, des discours qui diffusent et vulgarisent les connaissances dans un public extérieur aux cercles étroits de spécialistes : discours dits "secondaires ", qui reformulent les premiers et les commentent à l'usage de publics divers (spécialistes d'autres disciplines ou sous disciplines, personnes en formation initiale ou continue, amateurs de vulgarisation). 

une notion. Outre la définition du terme comme "désignation d'une notion ", on voit apparaître ici l'expression, qui a valeur de terme, langue de spécialité. La notion associée, peu appréciée par la plupart des linguistes, suggère que les discours spécialisés relèveraient d'un autre système linguistique que celui de la langue naturelle de leurs auteurs. Cela heurte l'intuition et l'expérience de tout locuteur, qui reconnaît sans hésitation, dans un texte scientifique ou technique non formalisé, de l'anglais ou du français par exemple. En général, les utilisateurs de l'expression langue de spécialité visent certaines caractéristiques rhétoriques, dont l'usage spécialisé de termes, qui s'observent dans les textes, ou discours, spécialisés. Quant au code utilisé par bien des spécialistes, incluant divers symboles étrangers à la langue naturelle, on les considère généralement comme des «langages». On peut dire que les discours spécialisés utilisent à la fois un code (langage artificiel fait de symboles) et une langue naturelle; de plus, ils peuvent intègrer des «images » diverses (schémas, graphiques, photographies...). Quoi qu'il en soit, pour désigner le vocabulaire « spécial » des discours en question, on préférera, avec Kocourek, parler de terminologie (ensemble de termes).

Quoi qu'il en soit, le terme, ici, apparaît défini à l'aide de deux critères, non indépendants : c'est la désignation d'une notion, ou, comme le disent A. Rey ou B. de 
Bessé, le nom d'une notion ; et ce nom est intégré à un ensemble d'autres noms de notion, en référence auxquels il est défini. On retrouve donc indirectement la référence à cet ensemble que constituent les discours spécialisés, pour définir les termes.

\section{La définition des termes dans les discours spécialisés}

61 On a affirmé plus haut que les définitions de termes se trouvent aussi bien dans les discours primaires que dans les discours secondaires. Leur fonctionnement n'y est cependant pas identique.

\section{L'institution des termes dans les discours primaires}

62 Si l'on comprend facilement pourquoi un spécialiste auteur d'un ouvrage de vulgarisation se croit obligé de définir certains termes, on peut s'étonner qu'un spécialiste, s'adressant à ses pairs, trouve utile de définir son vocabulaire. Pourquoi le fait-il ? La réponse est : parce qu'il l'invente.

63 C'est un phénomène récurrent dans les textes primaires que la définition d'un terme nouveau par un chercheur qui propose un nouveau concept. Cette pratique, déjà théorisée par la Logique de Port-Royal, s'observe entre autres dans les Principes de Descartes présentant son concept de tourbillon. C'est dans ce type de texte que se révèle le plus clairement la propriété qu'a le terme de nommer un concept. Et je me contenterai ici de rappeler l'exemple magistral de synapsie, tout particulièrement explicité par $\mathrm{E}$. Benveniste, qui écrit dans « Formes nouvelles de la composition nominale en français » :

Pour désigner les grandes unités et consacrer le phénomène spécifique qu'elles représentent, un terme nouveau devient nécessaire. (Benveniste 1966, repris dans en 1974, p.172).

64 Les exemples sont légion, et les caractéristiques des discours concernés sont assez stables: description du phénomène, prise en charge le plus souvent explicite de la néologie (sémantique ou formelle) par l'énonciateur. (Mortureux 1988 et 1991).

Dans ce type d'emploi, l'originalité d'un terme, c'est d'abord qu'il s'agit d'un néologisme à vocation lexicalisable. L'auteur du néologisme, s'il en assume, et même en revendique la paternité, souhaite l'intégrer au vocabulaire de sa discipline, et le voir adopté par la communauté à laquelle il appartient; l'emploi, fréquent, du verbe "proposer», par exemple ci-dessous, en est la trace.

Ensuite, c'est sa distinctivité. Là encore, Benveniste est particulièrement clair, comme le montre la suite de la phrase citée ci-dessus :

...un terme nouveau devient nécessaire, qui soit distinct de « composition » (il s'agit précisément de quelque chose d'autre que la composition), distinct aussi de "syntagme ", pour laisser à «syntagme " sa désignation propre qui s'applique à n'importe quel groupement, même occasionnel, opéré par des moyens syntaxiques, alors que nous avons ici une unité fixe. Nous proposons à cette fin un terme qui semble adéquat et clair... (o.c. p.172).

Les deux termes cités par Benveniste nomment les concepts relativement auxquels il situe l'originalité de la synapsie, qui, sans se confondre avec aucun d'eux, partage certains traits de chacun : comme la composition, elle crée des unités lexicale complexes à partir de bases, mais par des moyens syntaxiques, qui donnent à ses produits l'apparence de syntagmes. Le terme est bien le nom d'un concept défini, délimité par les concepts voisins dans le système auquel ils sont intégrés. 

interprétable; ainsi la phrase :

...un contact entre la matière de l'antimatière et la matière ordinaire de son environnement serait inévitable. (H. Fritzsch, o. c. p.276).

bien qu'elle soit extraite d'un ouvrage de vulgarisation, n'est guère compréhensible que dans le cadre théorique de la physique atomique, où sont définis les concepts de matière et d'antimatière. Car la "matière ordinaire » opposée à la "matière de l'antimatière " serait en quelque sorte la "matière de la matière ", ce qui n'a pas de sens dans le vocabulaire général. Dans le domaine de la physique, les particules et les antiparticules sont définies par leur symétrie, et le nom courant matière acquiert une acception spécialisée, terminologique, qui sert de base au terme dérivé antimatière; dans cet exemple, si antimatière est explicitement un terme, l'emploi de matière recèle un jeu sur la polysémie du lexème, qui associe acceptions générales et acception spécialisée.

$70 \mathrm{Ce}$ jeu sur la polysémie des lexèmes qui possèdent une ou plusieurs acceptions spécialisées est fréquemment observé dans la vulgarisation scientifique. Car la distinctivité originelle tend, au cours de la diffusion de plus en plus large du concept à travers des discours secondaires, à s'estomper. Au terme du processus de diffusion, la banalisation du terme peut se produire, aboutissant éventuellement à l'apparition ou au développement de la polysémie, à l'instar de budget.

71 Mais les discours primaires demeurent le lieu où s'élaborent les concepts que la terminologie s'applique à représenter en système, au sein desquels un terme est le nom d'un concept, ou d'une notion : les terminologues préfèrent souvent ce terme à celui de concept, plus utilisé par les linguistes; ce couple (notion/concept) permet peut-être de souligner la spécificité de l'analyse terminologique qui élabore un système de notions plus explicite que ne peut le faire la linguistique, lorsqu'elle parle de «système de signes ».

\section{La présentation des termes dans les discours secondaires}

Tous les discours secondaires comportent des définitions de termes. Elles peuvent affecter des formes très variées, ne répétant pas, en règle générale, la définition originelle, mais la reformulant. D'une part, elles présentent le terme comme préexistant, et non créé dans le discours; d'autre part, elles prétendent adapter son usage à un public non hautement spécialiste. Ainsi, dans des ouvrages de vulgarisation de la physique contemporaine cités ci-dessus, les deux auteurs se gardent bien de donner une définition explicite du terme neutrino lorsqu'ils le font apparaître dans leur discours :

La libération de l'énergie du vide dans l'univers [...] va donner naissance au contenu matériel de l'univers. [...]les neutrinos [...] vont surgir du vide. (Trinh Xuan Than, La mélodie secrète, Paris, Fayard, 1988, p.153).

Il y a d'ailleurs très vraisemblablement dans la nature, mis à part les photons, [...] d'autres particules qui se meuvent toujours à la vitesse de la lumière, les neutrinos. Ce sont des particules électriquement neutres, apparentées aux électrons. Ce sont en quelque sorte les frères neutres de l'électron, créés au cours de certaines réactions nucléaires. [...] "très vraisemblablement» [...] parce qu'on ne sait toujours pas, à ce jour, si ces neutrinos, comme les photons, n'ont pas de masse mais une énergie déterminée, ou s'ils en possèdent une, même très petite. Dans ce dernier cas, ils ne peuvent jamais se déplacer à la vitesse de la lumière, à l'instar des protons du CERN. (Harald Fritsch, $\mathrm{E}=\mathrm{mc}^{2}$ une formule change le monde, trad. Paris, $O$. Jacob, 1998, éd. originale Piper Verlag, Munich, 1988). 
Dans le premier cas, il n'y a pas de définition explicite; on déduit seulement que les neutrinos sont de la matière, au même titre que les autres particules évoquées. Dans le second, les informations, plus nombreuses, qui sont données, bien qu'elles contiennent en puissance une définition, ne revêtent pas la forme rhétorique définitoire: elles sont intégrées au reste du discours, et emploient des vocables étrangers à la terminologie physique, par exemple « frères ».

\section{Lexiques spécialisés et terminologies}

74 L'ensemble des termes est enregistré dans des recueils spécialisés, terminologies ou «lexiques", ceux-ci fréquemment annexés aux discours secondaires (ouvrages didactiques ou vulgarisateurs).

\section{L'élaboration des terminologies}

B. de Bessé définit ainsi les terminologies :

Les terminologies, c'est-à-dire l'ensemble des termes appartenant à un domaine, sont de véritables systèmes définitionnels. Elles reflètent l'organisation structurée d'un domaine bien délimité. Les termes sont organisés en systèmes structurés reflétant une organisation conceptuelle. La définition, qu'elle soit terminologique ou terminographique, passe par une analyse du concept. Pour le terminologue, comme pour le terminographe, "définir ", c'est décrire, délimiter, distinguer des concepts. Leurs définitions sont classificatoires, hiérarchisantes, structurantes. (La définition, Paris, Larousse, 1990, pp. 252-261).

Ces propos insistent sur le caractère notionnel, lui-même lié à un domaine, de l'organisation de ces ouvrages. Ce principe d'organisation les ferme sur eux-mêmes, et simultanément oriente les définitions de termes vers la plus grande distinctivité possible au sein du système. Comme le rappelle encore B. de Bessé, citant A. Rey :

Au plan notionnel, pour qu'un nom ait droit au titre de terme, il faut qu'il puisse, en tant qu'élément d'un ensemble (une terminologie), être distingué de tout autre. (o.c. p. 253).

Nous avons pu observer ces caractéristiques dans l'extrait du document européen qui fixe la terminologie de la terminologie! Ordre adopté pour présenter les articles numérotés et définitions en référence aux termes précédemment définis systématisent et fixent la distinctivité des termes.

L'élaboration de tels documents comprend plusieurs étapes: la terminographie commence par relever les termes dans les discours primaires, et en consigne la ou les définition(s). Puis le travail proprement terminologique construit le système de notions et réécrit au besoin la définition de chaque terme en valorisant le système retenu, ce qui entraîne notamment la réduction maximale de la synonymie et de la polysémie. Dans ces documents terminologiques, l'ensemble des termes correspondant à un domaine se trouve ainsi rassemblé, structuré et fixé, de façon infiniment plus explicite que dans les discours primaires, qui, échangés entre pairs, font l'économie de bien des précisions. Nulle part ailleurs que dans les terminologies, les termes ne répondent mieux à la définition qui fait de chacun d'eux le «nom d'une notion ».

79 En France, l'activité terminologique donne lieu à une consécration officielle, via une série d'institutions, dont la cheville ouvrière est constituée par les Commissions officielles de terminologie. Les listes élaborées par elles, domaine par domaine, par des équipes mixtes 
associant linguistes et spécialistes du domaine, sont sanctionnées par la publication au Journal officiel, ce qui leur donne force de loi. Car l'activité terminologique est le plus souvent déterminée par des fins pragmatiques, au premier rang desquelles figurent aujourd'hui les contacts internationaux entre spécialistes ; les terminologies sont donc en règle générale plurilingues.

\section{Glossaires}

Les listes de termes appartenant à un domaine particulier ont en général une visée étroitement didactique. Parfois indépendantes (vocabulaires juridique, informatique, ...), souvent liées à un ouvrage déterminé, elle présentent des définitions des termes considérés comme essentiels pour la compréhension d'un domaine ou d'un ouvrage. Le niveau de spécialisation de l'ouvrage, lui-même lié au public visé, détermine la teneur de la définition, plus ou moins spécialisée, plus ou moins dépendante de l'ensemble des concepts du domaine; c'est la raison pour laquelle on observe couramment, pour un même terme dans un domaine, des définitions non identiques (Mortureux 1987). L'analyse de ces paradigmes définitionnels éclaire sur la conceptualisation des phénomènes.

81 Les deux ouvrages de vulgarisation cités plus haut comportent chacun une de ces listes, qui définissent ainsi neutrino:

Neutrino : particule neutre sujette uniquement à la force nucléaire faible et, si elle possède une masse, à la force gravitationnelle. Produits en grand nombre dans les premiers moments de l'univers et en moindre nombre au coeur des étoiles et dans les supernovae, les neutrinos peuvent dominer la masse de l'univers si leur masse est égale au millionième de celle de l'électron. Avec une masse d'un dixième de millième de celle de l'électron, leur gravité peut arrêter l'expansion de l'univers et le faire s'effondrer sur lui-même. On ne sait pas à l'heure actuelle si le neutrino possède une masse. (Trinh Xuan Thuan, La mélodie secrète, $p .365$ ).

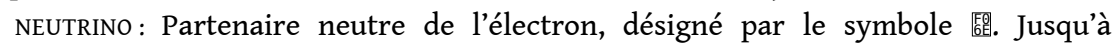
présent, on a établi l'existence de trois neutrinos différents: le neutrino électronique, le neutrino 監 et le neutrino 讶. (H. Fritzsch, $\mathrm{E}=\mathrm{mc}^{2}$ Une formule change le monde, p. 297).

82 On peut constater la variabilité des informations proposées pour le même terme par les trois ouvrages cités (dictionnaire de langue, et ouvrages de vulgarisation écrits par des spécialistes). En dehors du trait spécifique « neutre » qui est commun aux trois, et renvoie à la motivation du signe, on observe une variation sur l'incluant ("particule/ partenaire ») et sur le trait spécifique relatif à la masse : présent dans le PRE et l'un des lexiques spécialisés, il est absent de l'autre, qui seul énumère, en revanche, les trois sortes de neutrinos. On peut remarquer, en outre que dans chacun des deux ouvrages de vulgarisation, la richesse de la définition proposée dans le glossaire est inversement proportionnelle à la richesse des informations fournies lors de l'apparition du terme dans le fil du discours.

83 Au sein des collectivités de spécialistes, la maîtrise de la terminologie va de pair avec la maîtrise du domaine. Son apprentissage accompagne la formation des futurs spécialistes, et il ne pose guère de problème spécifique ; car derrière le vocable, c'est le concept qui retient l'attention ; le terme est le nom d'un concept, et c'est la maîtrise des concepts qui est visée. Dans cette perspective, l'usage de la terminologie est bien ressenti comme une commodité. 


\section{L'illusion lexicale et le travail de la langue}

84 des termes. Mais dans une société développée comme la nôtre de très nombreux locuteurs sont à la fois des spécialistes dans leur domaine d'activité, et des «naïfs " ailleurs. On peut penser que cette situation suffirait à apaiser la polémique. Or, ce n'est pas vraiment le cas. Car la maitrise d'un certain vocabulaire (qui peut être ou non une terminologie) entraine chez les locuteurs concernés une illusion de naturalité à l'égard de ce vocabulaire. Combien d'enseignants de littérature s'alarment aujourd'hui de voir le vocabulaire classique méconnu ou inconnu de leurs élèves, dont ils déplorent alors la pauvreté lexicale, sans prendre en compte le vocabulaire contemporain beaucoup mieux maitrisé (Lebrun 1986). Chacun a donc tendance à trouver claire et indispensable la terminologie de sa propre spécialité, parce qu'il l'a assimilée, et à juger inutilement obscure celle des autres, parce qu'il l'ignore, ce qui le prive d'un accès immédiat aux spécialités des autres: est-ce une trace transmise par l'école de l'idéologie sociolinguistique du XVIIème siècle, ou une simple réaction psychologique assez compréhensible? Quoi qu'il en soit, cela ne confère pas aux termes un statut lexical particulier.

S'agissant des termes, on sait aussi que beaucoup d'entre eux ont tendance à se banaliser. Comment s'effectue, du point de vue linguistique, cette opération? Au fur et à mesure de la diffusion des connaissances scientifiques et techniques, les termes qui leur correspondent se répandent dans des discours de plus en plus éloignés des sources primaires. Il y désignent des objets ou des processus qui relèvent de l'expérience courante. Par exemple, un carburateur de voiture, qui a dans la terminologie de l'industrie automobile une stricte définition, désigne un objet dont tout automobiliste a une certaine expérience; si la plupart des automobilistes peuvent désigner cet objet sous le capot, combien sauraient définir le terme de façon à satisfaire un spécialiste de mécanique automobile? Dans le sens lexical, la fonction désignative du vocable dans un discours donné peut n'actualiser que très pauvrement la signification du lexème sans dévier ou bloquer la communication. De même, l'application du terme synapsie à des séquences $\mathrm{N}$ de $\mathrm{N}, \mathrm{N}$ à N, N Adj ne s'accompagne pas nécessairement, pour les récepteurs, de la mise en contraste de ces formations avec d'autres, relevant des concepts "composition " et « syntagme »; couramment (par exemple dans les productions d'étudiants aux concours de recrutement) la synapsie est une variété de mot composé, ce qui n'est pas faux, mais manque de précision. La distinctivité de la signification d'un terme, liée au système de concepts du domaine concerné, s'efface au profit de son efficacité dans la désignation, au fur et à mesure que son emploi se répand en dehors des cercles de spécialistes. Et cela à l'insu même des locuteurs qui banalisent le terme sans en avoir conscience. Preuve supplémentaire, s'il en était besoin, que les termes sont des mots comme les autres, intégrés au lexique de la langue, dont ils partagent les propriétés. Leur actualisation dans des discours divers joue diversement sur la relation entre signification et désignation. Mais, contrairement à ce qu'on a parfois suggéré, les termes ne cessent pas d'être termes en changeant de discours, même si certains locuteurs ne les perçoivent pas comme tels. Plus que bien d'autres vocables, ils se prêtent à des interprétations différenciées, plus ou moins proches de la définition d'origine, en fonction du type de discours, et du niveau de 
formation des récepteurs. C'est la diversité des locuteurs qui crée la question des termes, plus que les termes eux-mêmes; et l'approche ne peut en être que sociolinguistique.

\section{BIBLIOGRAPHIE}

BENVENISTE E. : Problèmes de linguistique générale, Paris, Gallimard, 2 tomes, 1966 et 1974.

CHAURAND J. et MAZIÈRE Fr. éd. : La définition, Paris, Larousse, 1990).

DELESALLE S. et REY A. : «Problèmes et conflits lexicographiques », Langue française $n^{\circ} 43$, pp. 4-26.

KOCOUREK R. : La langue française de la technique et de la science, Wiesbaden et Paris (la documentation française), 1982.

LEBRUN Cl. : 1000 mots pour réussir, Paris, Belin, 1986 (Guide pédagogique fondé sur une enquête portant sur la compétence lexicale des élèves à l'entrée en seconde, non publiée en tant que telle).

MORTUREUX M.-Fr. (1979) : " Note à propos de Dictionnaire encyclopédique et vulgarisation ", Néologie et lexicologie, Paris, Larousse, 1979, pp. 192-198.

MORTUREUX M.-Fr. (1987) : «L'ambiguïté dans les paraphrases de termes scientifiques », L'ambiguité et la paraphrase, Centre de publications de l’Université de Caen, 1987, pp. 293-298.

MORTUREUX M.-Fr. (1988) : « Vocabulaire scientifique et circulation du savoir », Protée, vol 16, nº 3, pp. 99-105.

MORTUREUX M.-Fr. (1991) : « Néologie lexicale et énonciation personnelle dans le discours scientifique », Linx $\mathrm{n}^{\circ}$ spécial, pp. 71-83.

MORTUREUX M.-Fr. (1995) : « Les vocabulaires scientifiques et techniques », Carnets du Cediscor , n³, pp. 13-25.

MORTUREUX M.-Fr. (1997) : La lexicologie entre langue et discours, Paris, SEDES.

Dictionnaires :

Le Petit Robert (PR), 1966.

Le Petit Robert Electronique (PRE), 1996.

Lexis, Larousse, 1979.

\section{NOTES}

1. Texte reproduit tel qu'édité par la revue 


\section{RÉSUMÉS}

$\mathrm{Fr}$

Les termes constituent-ils une catégorie particulière de mots? D'une part, l'observation de l'expérience courante des locuteurs, et du traitement des termes dans les dictionnaires de langue généraux ne conduit pas à faire d'eux une catégorie homogène de mots, distincts du reste du vocabulaire. D'autre part, si l'on se tourne vers un usage et une représentation spécialisés des termes, ceux-ci semblent au contraire s'opposer par leur fonctionnement discursif au reste du vocabulaire. On en conclut que le statut des termes relève d'approches sociolinguistiques, leur statut lexical n'étant pas fondamentalement différent de celui des autres lexèmes.

Are the terms a special word's class? First, we can observe speaker's usual experiences, and term's representations in general dictionaries ; so terms are not word's homogen class, separate from other words. Secondly, we can observe term's specialised uses and representations; so terms appears different from other words. Consequently, their lexical status is not different from other lexems, but their difference is sociolinguistical.

\section{AUTEUR}

\section{MARIE-FRANÇOISE MORTUREUX}

Professeur émérite à l'Université Paris X 